\title{
UNE CINQUIÈME FILAIRE DU GENRE DIPETALONEMA, PARASITE DE SINGES SUD-AMÉRICAINS
}

\author{
O. 13AIN*, M. IIAGNI*, IR. MLLILEI**
}

RÉSUMÉ. Description de D. Jreitasi n. sp., parasite de Cebus capucinus; l'espèce, proche de D. caudispina, s'en distingue principalement par le vagin et le spicule droit un peu plus complexes la microfilaire plus courte, l'area rugosa non prolongée sur la queue.

Compléments morphologiques sur le måle D. robini Petit et coll., 1985.

Tableau comparat if de 4 principaux caractères évolutifs che\% les Dipetalonema (vagin, spicules gauche et droit, fourreau épithélio-musculaire (lu mâle) : $D$. caudispina el $D$. /reitasi n. sp. forment un petit groupe primitif qui s'oppose à l'ensemble plus évolué, I. robini, D. gracile et D. graciliformis.

Mots-clés : Morphologie. Taxonomie. I'ilarioidea. Onchocercidae. Primates. P'latyrhiniens

\section{A fifth filaria of the genus Dipetalonema, parasite of South-American monkeys.}

SUMMARY. Description of $D$. freitusi n. sp., a parasite of Cebus capucinus : I his species, close 10 D. caudispina, is distinguished by the slightly more complex structure of the vagina and right spicule, the lack of area rugosa on the tail and by the shorter microfilaria.

Additional morphological data on the male of $D$. robini petil et al., 1985 are given.

A comparative table of 4 principal evolutive characters of the genus IDipetalonema (vagina, left and right spicules, epithelio-muscular body wall) is given. $D$. caudispina and $D$. (reitasi $\mathbf{n}$. sp. represent a small primilive group opposed to the more evolved one made up of $I$ ). robini, $D$. gracile and D. graciliformis.

Key-words: Morphology. Taxonomy. liblarioidea. Onchocercidae. Primates. Platyrhinies.

Au cours des identifications de routine, les Filaires de Singes sud-américains du genre Dipelalonema sont habituellement rapportées à l'une des trois espèces classiquement connues : D. caudispina (Molin, 1858), D. gracile (Rudolphi, 1819) ou D. graciliformis (Freitas, 1964).

Or, la récente mise en évidence d'une quatrième espèce, $D$. robini Petit

* Laboratoire de Zoologie Vers, 61, rue de Buffon, F 75231 Paris Cedex 05.

* C. A. B. International Institute of Parasitology, 39; a Hatfield Road, St. Albans $A L$ t OXU, U. K.

Accepté le 30 janvier 1987. 
et coll., 1985, suggère que la diversité des Dipetalonema est plus grande qu'on ne le pensait.

Cette notion est confirmée par la nouvelle espèce décrite ici.

Le matériel étudié provient d'un Cebus capucinus mort au Jardin Zoologique de Londres en 1952 ; il comprend 7 femelles et 3 mâles. Les types sont conservés dans les collections du LSHTM, Londres $\left(n^{\circ} 512\right)$ et les paratypes au M. N. H. N., Paris ( ${ }^{\circ} 127$ ED).

\section{Description de D. freitasi n. sp.}

Les principaux caractères morphologiques sont représentés sur les figures $1,2,3$.

Quelques précisions complémentaires sont données sur les papilles de la tête, la structure du vagin et celle du spicule droit.

- Papilles de la tête : chez la femelle, indices labial et céphalique (cf. Bain et coll., 1985) respectivement de +33 et +26 ; chez le mâle, indices labial et céphalique respectivement de +15 et +25 .

-- Vagin ( fig. $1 \mathrm{~F}$ el $\mathrm{G}$ ) : les deux spécimens figurés diffèrent par la longueur du vagina vera et la taille des cellules épithéliales. D’après des observations faites sur une autre Filaire (Bain et coll., 1985), ces divergences semblent être liées au degré de maturité des femelles, $F$ représentant une forme plus jeune que $G$.

- Spicule droit : sa région distale a la morphologie suivante : l'aile membraneuse droite, soutenue par un axe plus cuticularisé courbé vers la face ventrale, forme l'extrémité dı spicule constituée par une pointe bifide terminale et, sur la face interne gauche, une lame enroulée en cornet. L'aile membianeuse gauche s'interrompt brusquement à une trentaine de $\mu \mathrm{m}$ de l'apex, quand elle a sa largeur maximale ; sous certains angles, le bord terminal de cette aile apparaît si nettement que l'extrémité distale du spicule semble articulée sur le corps du spicule (fig. $3 \mathrm{~A}$ ì $D)$.

\section{DIMLNSIONS}

-- Femelle holotype : corps long de $230 \mathrm{~mm}$, large de $460 \mu \mathrm{m}$; anneau nerveux à $230 \mu \mathrm{m}$ de l'apex ; cavité buccale haute de $10 \mu \mathrm{m}$; œesophage long de $2000 \mu \mathrm{m}$ avec portion musculaire longue de $425 \mu \mathrm{m}$; vulve à $670 \mu \mathrm{m}$ de l'apex; vagin long de $390 \mu \mathrm{m}$ et large de $80 \mu \mathrm{m}$; ovéjecteur long de $8750 \mu \mathrm{m}$; queue longue de $510 \mu \mathrm{m}$; pointes caudales latérales longues de $10 \mu \mathrm{m}$.

- Une femelle paratype : corps long de $210 \mathrm{~mm}$, large de $450 \mu \mathrm{m}$; tête de $72 \mu \mathrm{m}$ et $54 \mu \mathrm{m}$ de côtés; anneau nerveux à $230 \mu \mathrm{m}$ de l'apex ; cavité buccale haute de $11 \mu \mathrm{m}$; œsophage long de $2040 \mu \mathrm{m}$ et portion musculaire longue de $440 \mu \mathrm{m}$; vulve à $650 \mu \mathrm{m}$ de l'apex ; vagin long de $380 \mu \mathrm{m}$ et large de $100 \mu \mathrm{m}$; ovéjecteur long de $8700 \mu \mathrm{m}$; queue longue de $590 \mu \mathrm{m}$; languettes caudales latérales et pointe terminale longues respectivement de $9-10 \mu \mathrm{m}$ et $6 \mu \mathrm{m}$; phasmides écartées de $30 \mu \mathrm{m}$ et situées à $34 \mu \mathrm{m}$ de l'extrémité caudale. Microfilaires utérines longues de 100 à $107 \mu \mathrm{m}$ et larges de $3,5 \mu \mathrm{m}$ à $4 \mu \mathrm{m}$. 


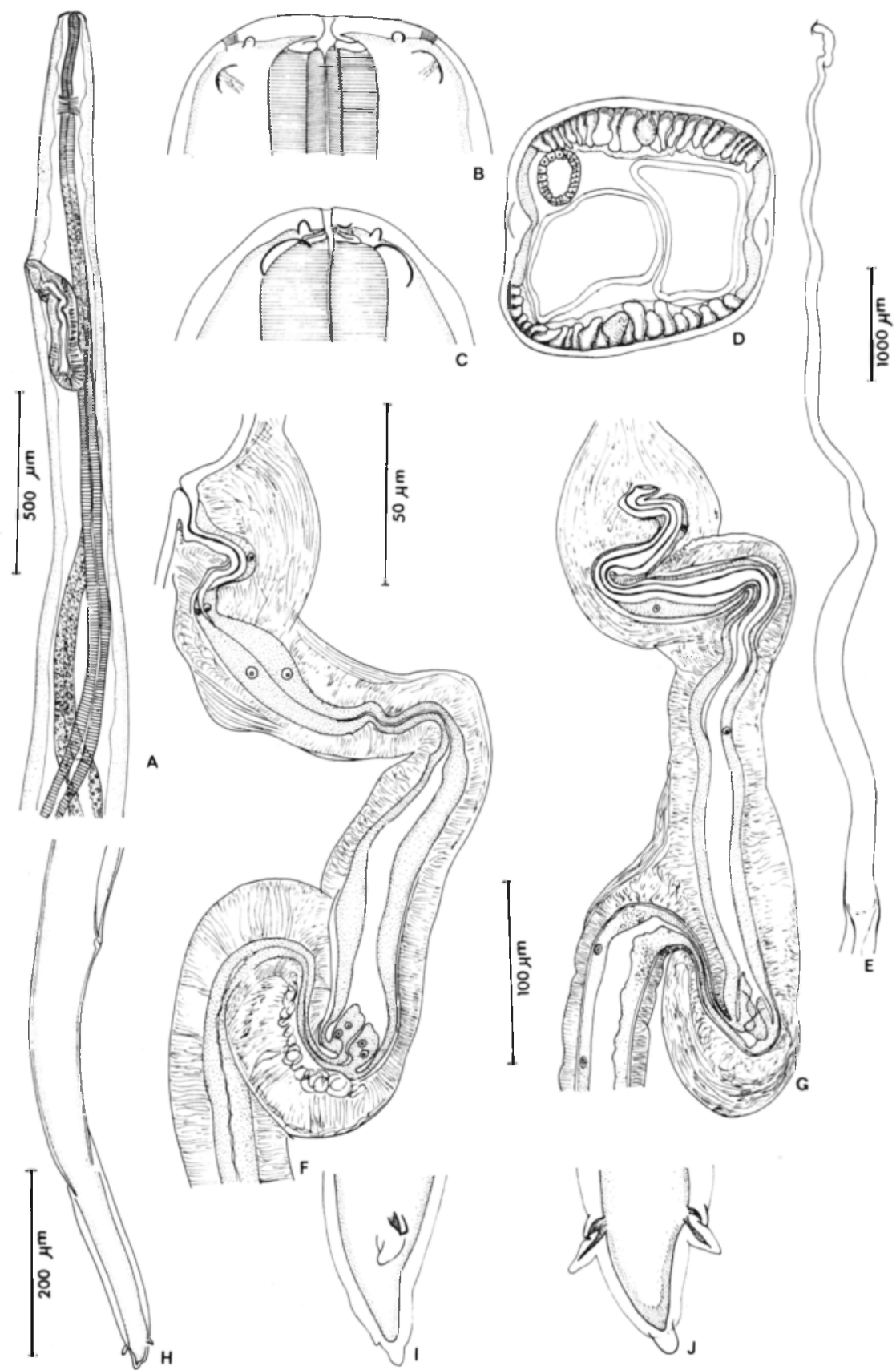

Fıg. 1. - Dipetalonema freilasi n. sp., femelle. A : région antérieure, vue latérale ; B et C : tête, vues médiane et latérale $; \mathrm{D}$ : coupe transversale à mi-corps ; $\mathrm{E}$ : ovéjecteur ; $\mathrm{F}$ et $\mathrm{G}$ : vagin, vulve en vues latérale et ventrale, chez 2 femelles; H : queue ; I et J : extrémité caudale, vues latérale (face ventrale à droite) et ventrale (Á éch. $500 \mu \mathrm{m} ; \mathrm{B}, \mathrm{C}, \mathrm{I}, \mathrm{J}$, éch. $50 \mu \mathrm{m}$; $\mathrm{D}, \mathrm{H}$, éch. $200 \mu \mathrm{m} ; \mathrm{E}$, éch. $1000 \mu \mathrm{m} ; \mathrm{F}, \mathrm{G}$, éch. $100 \mu \mathrm{m})$. 


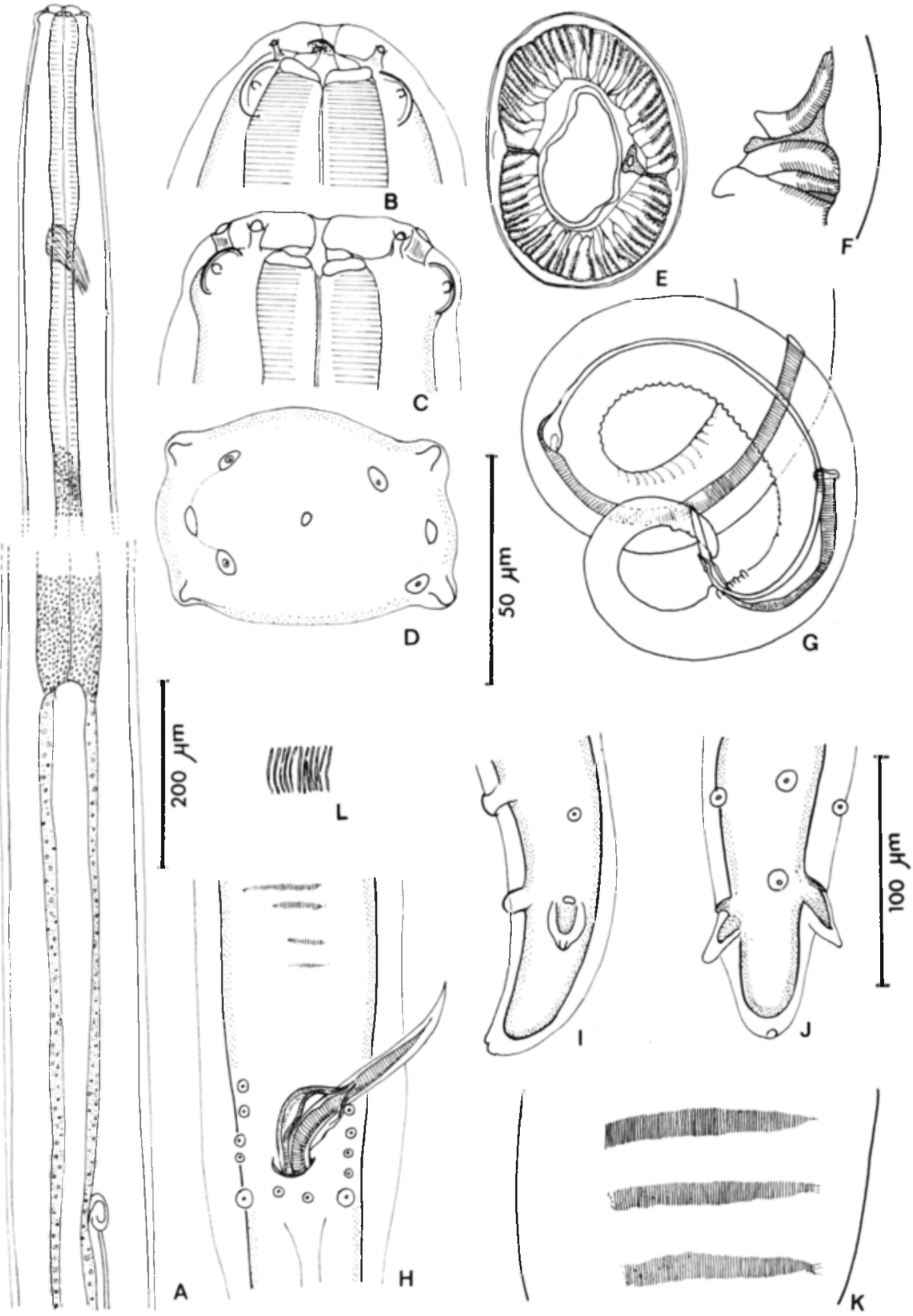

FIG. 2. - Dipetalonema freitasi n. sp., mâle. A : région céphalique et jonction cesophage-intestin, vue latérale; $\mathrm{B}, \mathrm{C}, \mathrm{D}$ : tête, vues latérale, médiane et apicale $; \mathrm{E}$ : coupe transversale à mi-corps; $\mathrm{F}$ : idem, détail de la corde latérale et des cellules musculaires voísines ; $\mathrm{G}$ : région caudale ; $H$ : région cloacale, vue ventrale; I et $J$ : extrémité caudale, vues latérale et ventrale ; IV : area rugosa, à mi-longueur de l'organe, vue ventrale; $\mathrm{L}$ : idem, détail d'une bande de bâtonnets (A, E, G, éch. $200 \mu \mathrm{m} ; \mathrm{B}, \mathrm{C}, \mathrm{D}, \mathrm{I}, \mathrm{J}, \mathrm{L}$, éch. $50 \mu \mathrm{m} ; \mathrm{F}, \mathrm{H}, \mathrm{K}$, éch. $100 \mu \mathrm{m}$ ). 


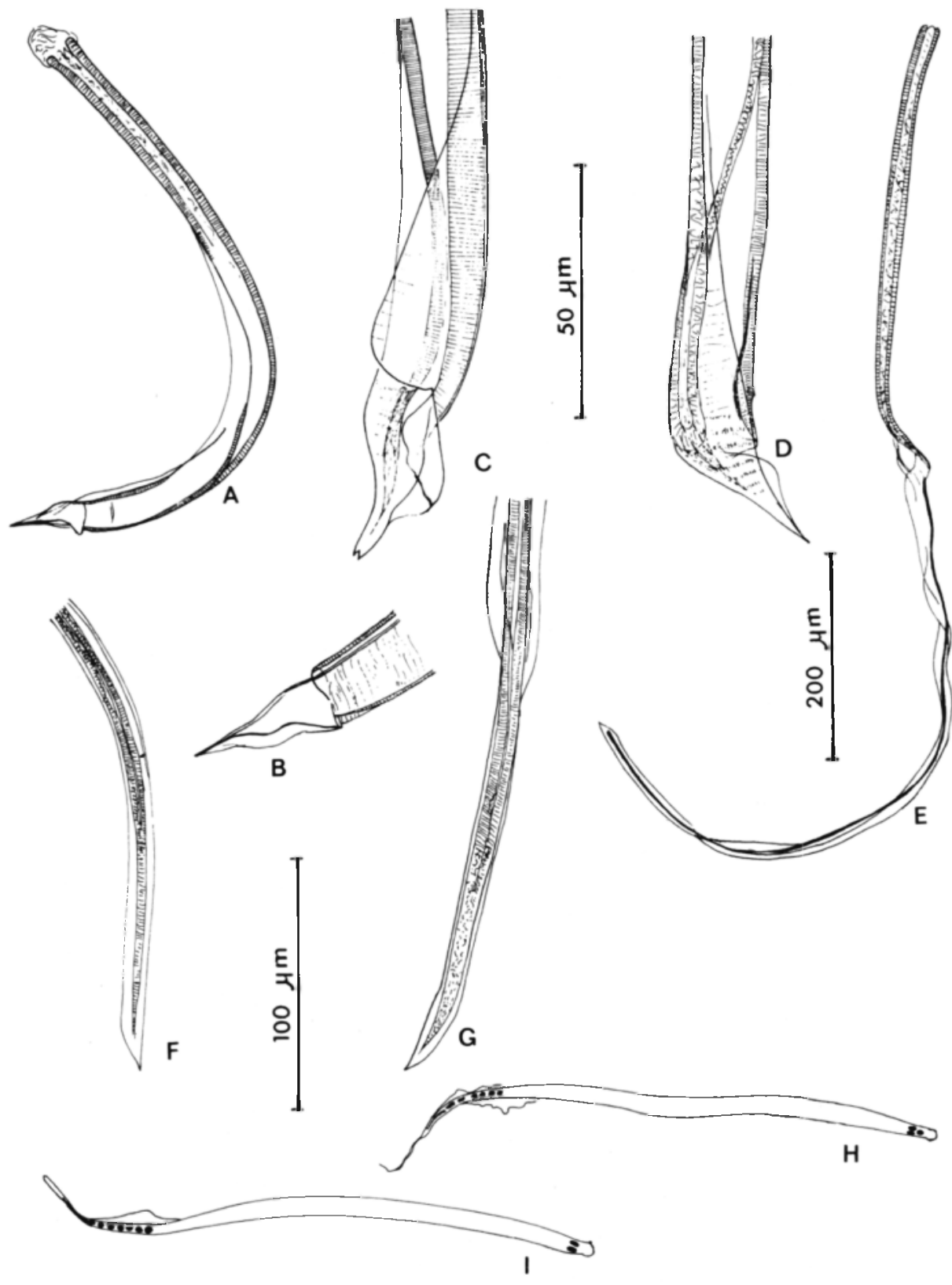

FIG. 3. - Dipetalonema freitasi n. sp. A à D : spicule droit ; A : vue latérale gauche ; B : idem, extrémité distale ; $\mathrm{C}$ : idem, vue ventrale ; $\mathrm{D}:$ idem, vue latérale droite ; $\mathrm{E}$ : spicule gauche, vue latérale gauche ; F : idem, extrémité distale, vue latérale gauche ; G : idem, vue ventrale ; $H$ et $I$ : 2 microfilaires utérines, respectivement crochet vu de profil et de face $(A, F, G$, éch. $100 \mu \mathrm{m} ; \mathrm{B}, \mathrm{C}, \mathrm{D}, \mathrm{H}$, I, éch. $50 \mu \mathrm{m}$; E, éch. $200 \mu \mathrm{m}$ ). 
- Mâle allotype : corps long de $100 \mathrm{~mm}$ et large de $260 \mu \mathrm{m}$; anneau nerveux à $260 \mu \mathrm{m}$ de l'apex ; cavité buccale haute de $13 \mu \mathrm{m}$; œsophage long de $1700 \mu \mathrm{m}$ et portion musculaire longue de $455 \mu \mathrm{m}$; queue longue de $325 \mu \mathrm{m}$; spicule gauche long de $985 \mu \mathrm{m}$ avec un manche long de $430 \mu \mathrm{m}$; spicule droit long de $250 \mu \mathrm{m}$.

— Un mâle paratype : corps long de $78 \mathrm{~mm}$ et large de $255 \mu \mathrm{m}$; tête de $60 \mu \mathrm{m}$ et $43 \mu \mathrm{m}$ de côtés ; anneau nerveux à $215 \mu \mathrm{m}$ de l'apex ; cavité buccale haute de $10 \mu \mathrm{m}$; cesophage long de $2100 \mu \mathrm{m}$ et portion musculaire longue de $455 \mu \mathrm{m}$; queue longue de $360 \mu \mathrm{m}$; spicule gauche long de $1040 \mu \mathrm{m}$, à manche long de $400 \mu \mathrm{m}$ et ailes de la lame interrompues à $150 \mu \mathrm{m}$ de l'extrémité distale; spicule droit long de $240 \mu \mathrm{m}$; gubernaculum long de $35 \mu \mathrm{m}$; area rugosa longue de $8000 \mu \mathrm{m}$; languettes caudales latérales longues de $10 \mu \mathrm{m}$; phasmides écartées de $23 \mu \mathrm{m}$ et situées à $35 \mu \mathrm{m}$ de l'extrémité caudale.

\section{Discussion}

Les trois espèces classiques de Dipetalonema ont été redécrites récemment (Bain et coll., 1986).

Par la silhouette de la région antérieure de la femelle (présence d'un rétrécissement post-vulvaire), la structure assez simple du vagin, le spicule gauche sans flagelle terminal, le spicule droit dépourvu de corolle plissée terminale et la microfilaire avec un léger cou, nos spécimens se rapprochent de Dipetalonema caudispina (Molin, 1859).

Toutefois ils se distinguent par la taille plus réduite du corps, l'œesophage plus court, la microfilaire presque deux fois plus courte, l'absence d'area rugosa sur la queue, le vagin quatre fois coudé, la présence de petites languettes phasmidiales chez la femelle, la position des papilles terminales du mâle, l'extrémité distale du spicule droit, moins simple, avec un bord enroulé en cornet et une petite pointe bifide.

Ce matériel, parasite de Cebus capucinus, représente donc une espèce nouvelle que nous nommons Dipetalonema freitasi n. sp., en hommage à J. F. Texeira de Freitas.

\section{Compléments morphologiques sur le mâle de $D$. robini, Petit et coll., 1985}

Le spécimen étudié est le mâle $226 \mathrm{NE}$ (dimensions in Petit et coll., 1985).

Fourreau épithéliomusculaire ( $f i g .4 \mathrm{~A}$ ) : la musculature de chaque quadrant comprend un groupe de cellules musculaires très riches en fibrilles et hautes, situé près de la corde latérale, et un groupe de cellules musculaires moins fibrillaires et moins hautes, situé près de la corde médiane.

Spicule gauche (fig. $4 B, C, D$ ) : la lame est bordée par 2 ailes à larges plis, qui se rabattent l'une vers l'autre et rejoignent leurs bords; elles s'interrompent 
brusquement à mi-longueur de la lame ; celle-ci se termine en flagelle ; l'extrémité distale est spatulée.

Spicule droit (fig. $4 E, F, G$ ) : lame en gouttière, bordée par 2 ailes et dont le fond cuticularisé se courbe en $S$ à l'extrémité distale ; cette partie terminale est entourée d'un large feuillet membranaire non plissé, replié à l'apex.
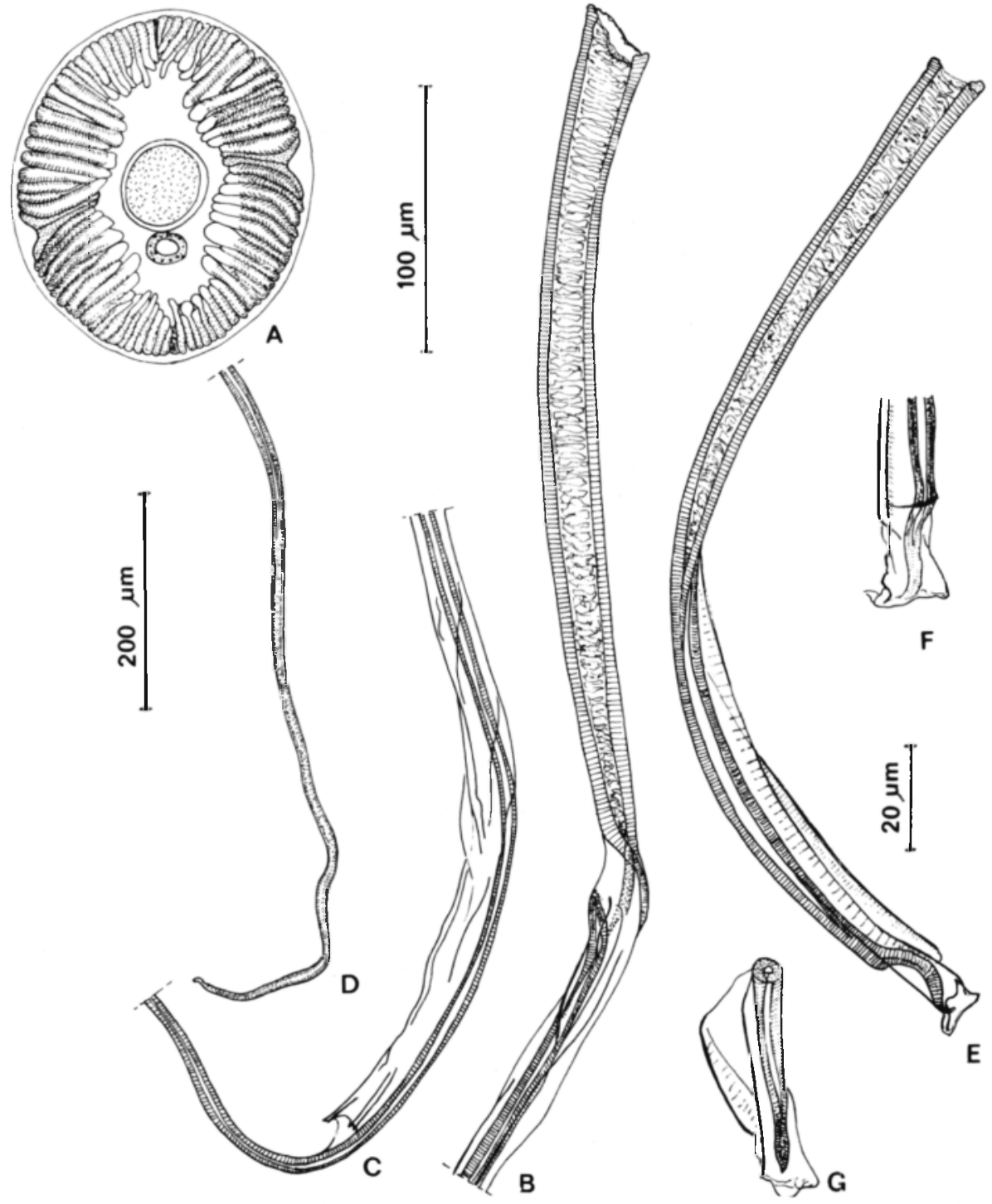

Fig. 4. - Dipetalonema robini Petit et coll., 1985, mâle. A : coupe transversale à mi-corps ; $\mathrm{B}, \mathrm{C}, \mathrm{D}$ : spicule gauche en trois morceaux, vue latérale ; $\mathrm{E}$ : spicule droit, vue latérale droite ; F : idem, extrémité distale, vue dorsale ; $\mathrm{G}$ : idem, mais sur spicule plié pour montrer l'aile gauche dilatée. 


\section{Conclusion}

Dunn et Lambrecht (1963), ainsi que Freitas (1964) ont signalé que les espèces de Dipetalonema présentaient des variations morphologiques suivant l'hôte parasité.

La mise en évidence de $D$. freitasi $n$. sp., proche de $D$. caudispina et celle de $D$. robini Petit et coll., 1985, proche de $D$. gracile, suggère en fait l'existence d'une grande diversité spécifique des Dipetalonema de Singes sud-américains.

Le petit nombre d'espèces connues et les données peu sûres sur leurs spectres

A

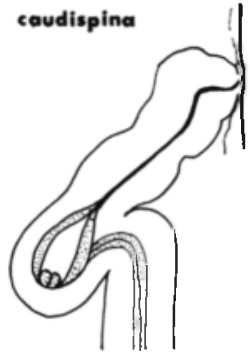

B

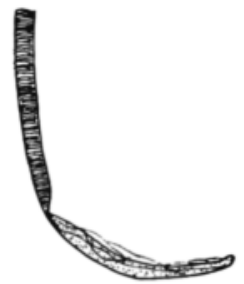

C

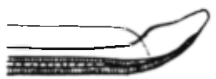

D

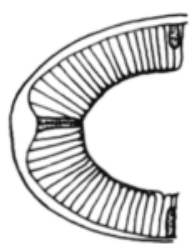

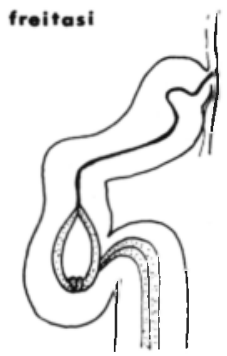

robini
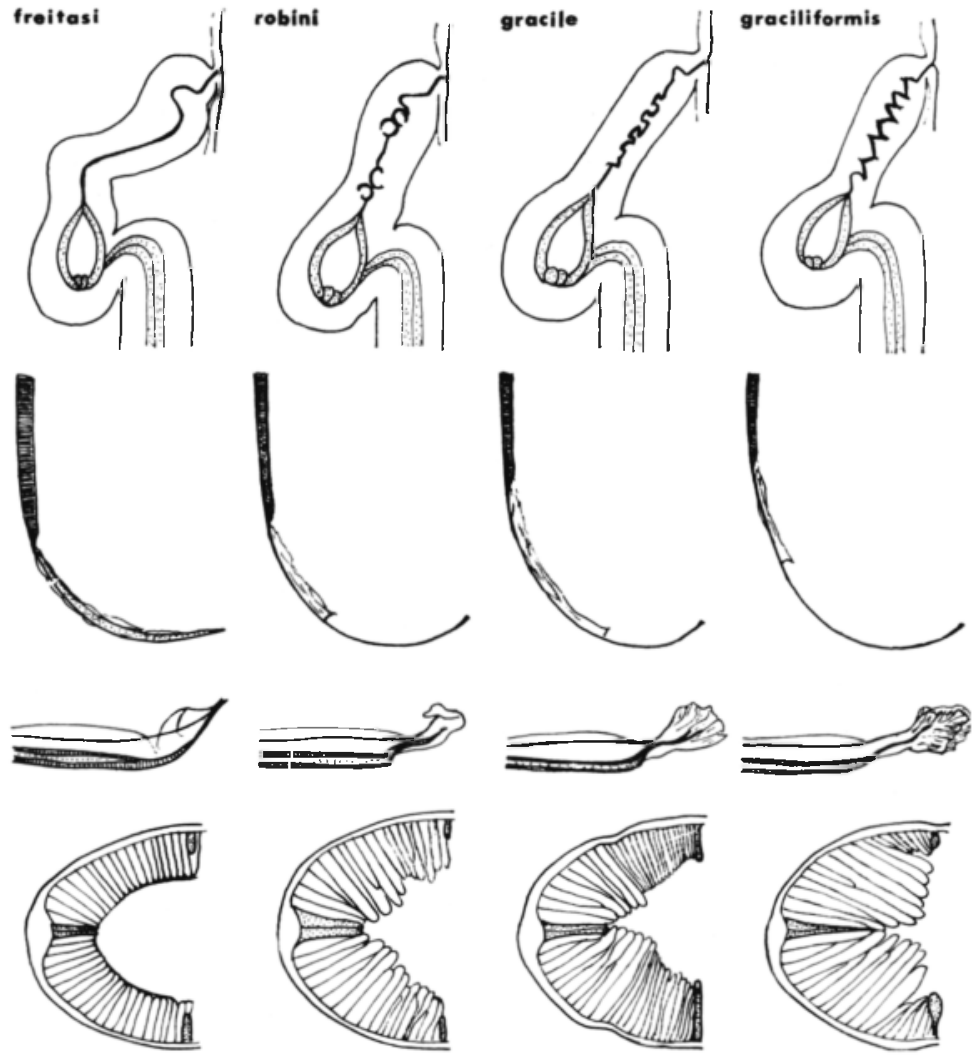

FIG. 5. - Représentation schématique de 4 caractères évolutifs chez les Dipetalonema. A, vagin : vagina vera presque rectiligne chez $D$. caudispina, coudé chez $D$. freilasi $\mathrm{n}$. sp., à 2 valvules chez $D$. robini, sinueux chez $D$. gracile et $D$. gracilliformis ; B, spicule gauche : manche aussi long que la lame chez $D$. caudispina, progressivement plus réduit chez les autres espèces; lame épaisse avec ailes atténuées dans la région distale chez $D$. caudispina et $D$. freitasi, mince et terminée en flagelle avec ailes interrompues brusquement loin de la pointe chez les 3 autres espèces ; $\mathrm{C}$, extrémité distale du spicule droit : extrémité simple chez $D$. caudispina, en cornet chez $D$. freitasi, en large feuillet replié, non plissé chez $D$. robini, en corolle plissée chez $D$. gracile et $D$. gracilliformis ; $\mathrm{D}$, fourreau épithéliomusculaire du mâle : musculature homogène chez $D$. caudispina et $D$. freitasi, hétérogène chez les 3 autres espèces; atrophie des cellules musculaires submédianes très marquée chez $D$. graciliformis. 
d'hôtes ne permettent pas actuellement une analyse de l'évolution du genre Dipetalonema en corrélation avec celle des Platyrhiniens.

On remarque seulement que $D$. caudispina, forme primitive par les caractères morphologiques larvaires et adultes (Bain et coll., 1986), constitue avec $D$. freitasi n. sp. un petit groupe qui s'oppose aux trois autres espèces morphologiquement plus évoluées, D. robini, D. gracile et D. graciliformis (fig. 5).

Comme $D$. caudispina, $D$. freitasi n. sp. est parasite de Platyrhiniens évolués, les Cebidae.

\section{BIBLIOGRAPHIE}

Bain O., Dissanaike A. S., Gross J. H., Harinasuta C., Sucharit S. : Morphologie de Wuchereria bancrofti adulte et sub-adulte. Ann. Parasitol. Hum. Comp., 1985, 60, 613-630.

Bain O., Petit G., Rosales-Loesener L. : Filaires de Singes sud-américains. Bull. Mus. nat. Hist. nat., Paris, $4^{\mathrm{e}}$ sér., 8,1986 , section $\mathrm{A}, \mathrm{n}^{\circ} 3,513-542$.

DunN F. L., LAmBRecrt F. L. : On some filarial parasites of south-american primates, with a description of Tetrapetalonema tamarinae n. sp. from peruvian tamarin marmoset. Tamarinus nigricollis (Spix, 1823). J. Helminth., 1963, 37, 261-286.

Freitas J. F. T. : Achegas Helminthologicas. R. Ci. Biol. Belèm, 1964, 2, 3-40.

Petit G., Bain O., Roussilhon G. : Deux nouvelles Filaires chez un Singe Saimiri sciureus au Guyana. Ann. Parasitol. Hum. Comp., 1985, 60, 65-81. 